\title{
Buckling criteria for vacuum bottle based on finite element analysis
}

\author{
K. Natori ${ }^{1}$, T. Kobayashi ${ }^{1}$, S. Tatsuta ${ }^{1}$, T. Tanaka ${ }^{2}$ \& Y. Arao ${ }^{2}$ \\ ${ }^{1}$ Graduate School of Engineering, Doshisha University, Kyoto, Japan \\ ${ }^{2}$ Faculty of Science and Engineering, Doshisha University, Kyoto, Japan
}

\begin{abstract}
A bottle with a vacuum insulation structure is generally called a thermos bottle. It has a dual structure with inner and outer cylinders and a vacuum layer between them. The bottle with the structure maintains the heat and cold. It has been found useful for many years as a container that carries drinking water. Recently, the lightweight design of a vacuum bottle has been required to meet consumer demand. However, the bottle's wall collapses during the process of drawing a vacuum if the stiffness of the cylinder is not enough. To construct the design guide that can prevent the collapse of a double-structured bottle induced by the pressure difference, a numerical simulation was performed. The critical thickness $t_{c}$, which is the thickness of the bottle when the bottle starts to deform under $1 \mathrm{~atm}$, was defined. $t_{\mathrm{c}}$ depends on the length and diameter of the bottle. The index to determine $t_{\mathrm{c}}$ from the length and diameter of the bottle was suggested. To evaluate the validity of the analysis, vacuum bottles were prepared and experiments were conducted. The index we suggested showed good agreement with the experimental results. The shift of index within the increase of Young's modulus can be formulated.
\end{abstract}

Keywords: light weight design, vacuum bottle, buckling, finite element method.

\section{Introduction}

In recent years, the public concern about environmental issues is growing and the attention to reusable bottles has increased. The well-known reusable bottle is a vacuum bottle (hereinafter simply called a bottle) which has a double-structured 
cylindrical wall as shown in Figure 1. There is a vacuum layer between the space of the inner cylinder and the outer cylinder. The space of the vacuum layer provides the high heat insulation property to the bottle.

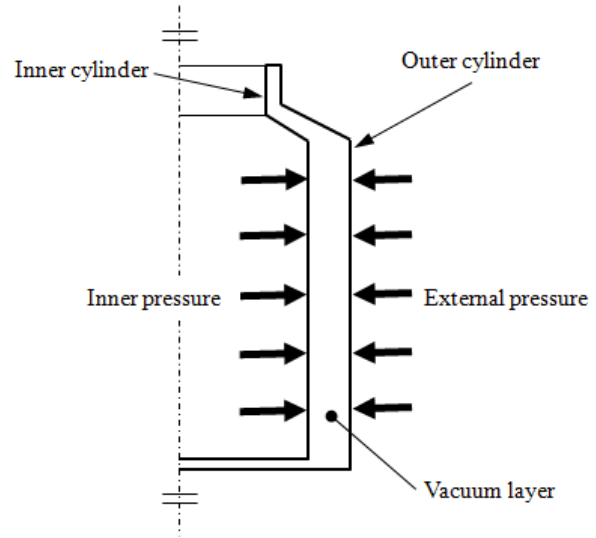

Figure 1: $\quad$ Cross-section of a vacuum bottle.

The weight saving of the bottle has been required by consumers. The benefits of weight saving of the bottles are not only for the convenience of carrying but also for the reduction of resource usage. In addition, the use of the reusable bottle can reduce the use of the disposable bottle, and contribute to the reduction of resource consumption. However, when the bottle's thickness is reduced for weight saving, the bottle's strength and stiffness also decrease. Problems such as cracks and wrinkles occur during the manufacturing process. The surface of the bottle is always subjected to atmospheric pressure. The most serious problem occurs when the bottle collapses during the process of drawing a vacuum when the bottle's thickness is not enough to maintain its shape under atmospheric pressure. The bottle should be designed to be as light weight as possible without the collapse, therefore an optimal design for the bottle might exist. However, the design of the bottle relies on know-how and experience, and there are no clear indicators in the design of the bottle. It is necessary to clarify the critical thickness to avoid an unexpected collapse during the manufacturing process of the vacuum bottle.

The purpose of this study is to suggest indicators that are necessary for the optimal design of the vacuum bottle. A finite element analysis was performed to predict the buckling strength of the bottle under the pressure load. The relationship between shape parameter (diameter, height, and thickness of bottle) and bucking pressure load was investigated based on a numerical simulation. Experiments were conducted to determine the buckling strength of the outer cylinder under the pressure load, and compared with the analytical results to investigate the validity of the analysis. 


\section{Analysis}

\subsection{Analytical model}

At first, the method for predicting buckling strength under pressure loading was introduced. In this study, we used commercial software of a non-linear structural analysis LS-DYNA ver.971 made by Livermore Software Technology Corp. In order to simulate the buckling strength of the bottle under pressure loading, double cylinder models based on actual products were created [1]. These models consist of outer cylinder body, outer cylinder bottom, inner cylinder body and inner cylinder bottom. The models are constructed using four nodes of shell elements. Total element number was between 4000 and 8000. Table 1 and Figure 2 show the geometry and the boundary condition of analytical models. $D_{1}$

Table 1: $\quad$ Dimension of a double cylinder model.

\begin{tabular}{|c|c|c|c|c|c|}
\hline \multicolumn{2}{|c|}{ Analytical model } & $D_{1}[\mathrm{~mm}]$ & $L_{1}[\mathrm{~mm}]$ & $D_{2}[\mathrm{~mm}]$ & $L_{2}[\mathrm{~mm}]$ \\
\hline \multirow{3}{*}{ B1 } & $\mathrm{a}$ & \multirow{3}{*}{56} & 106 & \multirow{3}{*}{52} & 88 \\
\hline & $\mathrm{b}$ & & 157 & & 136 \\
\hline & $\mathrm{c}$ & & 204 & & 183 \\
\hline \multirow{3}{*}{ B2 } & $\mathrm{a}$ & \multirow{3}{*}{60} & 160 & \multirow{3}{*}{56.6} & 131.65 \\
\hline & $\mathrm{b}$ & & 200 & & 171.15 \\
\hline & C & & 240 & & 211.65 \\
\hline \multirow{3}{*}{ B3 } & $\mathrm{a}$ & \multirow{3}{*}{67} & 120 & \multirow{3}{*}{62.4} & 84.65 \\
\hline & $\mathrm{b}$ & & 168 & & 133.65 \\
\hline & c & & 217 & & 182.65 \\
\hline
\end{tabular}

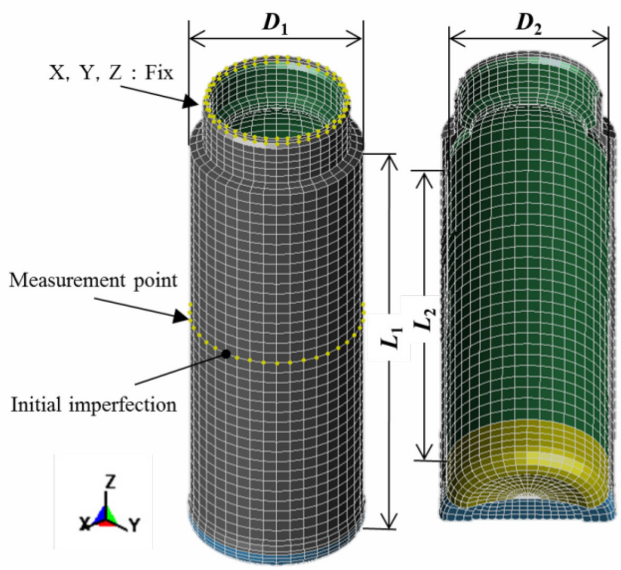

Figure 2: $\quad$ Geometry and boundary conditions of a double cylinder model. 
and $L_{1}$ are diameter and height of outer cylinder, respectively. $D_{2}$ and $L_{2}$ are those of the inner cylinder, respectively. $L_{1}$ and $L_{2}$ are heights of cylinder bodies excluding oral regions of the bottles. In order to discuss the effects of shape parameters on the buckling strength of the bottle, diameter and height are varied as shown in Table 1. Each thickness of inner and outer cylinder body is the same value. The thickness of the bottle varied from 0.1 to $0.35 \mathrm{~mm}$. The thickness of the bottom part is set a constant value of $0.4 \mathrm{~mm}$.

\subsection{Analytical condition}

In boundary condition, displacements of the nodes which are joining sections of the inner and outer cylinder were fixed in the direction of the $X, Y$ and $Z$ axes. It is assumed that the space between the inner and outer body in the double cylinder model was filled with fluid having a reference pressure condition, and additional pressure was loaded at $0.02-0.16 \mathrm{MPa} / \mathrm{sec}$ externally on the whole inner and outer cylinder. The difference of atmospheric pressure between the space and outer cylinder is used as a parameter that causes the collapse of the bottle. The volume elasticity of the fluid was set to $1.4 \times 10^{5} \mathrm{~Pa}$ and the mass density of it was $1.293 \mathrm{~kg} / \mathrm{m}^{3}$. Moreover, the initial imperfection is applied to the outer body of the analytical model as a starting point of deformation [2]. In initial imperfection, the coordinates of the point were $\left(x=D_{1} / 2, y=0, z=L_{1} / 2\right)$ and displacement of $-0.01 \mathrm{~mm}$ in the $X$ axial direction was given at one node. In order to check the deformation of the model, the amount of displacement at the node shown in Figure 2 was outputted. Table 2 shows the mechanical properties of material used in this analysis. These parameters were obtained by quasi-static tensile test using the same material with the experiments described in the next section. A material is assumed to be a perfectly elastic plastic solid.

Table 2: $\quad$ Material property in analysis.

\begin{tabular}{ccccc}
\hline Metal & $\begin{array}{c}\text { Mass } \\
\text { density } \\
\rho\left[\mathrm{g} / \mathrm{cm}^{3}\right]\end{array}$ & $\begin{array}{c}\text { Young's modulus } \\
E[\mathrm{GPa}]\end{array}$ & $\begin{array}{c}\text { Yield stress } \\
\sigma[\mathrm{MPa}]\end{array}$ & Poisson ratio \\
\hline SUS304 & 8.03 & 197 & 205 & 0.3 \\
\hline
\end{tabular}

\section{Experiment}

\subsection{Specimen}

To discuss the validity of the analysis, experiments were conducted. SUS304, which is austenitic stainless steel, was used for test material. First, the plate with a thickness of $0.2-0.4 \mathrm{~mm}$ was processed into steel pipe with $42-50 \mathrm{~mm}$ in diameter by welding technique. Then bulge forming, which is one of the manufacturing processes of the bottles, was conducted to expand and form the bottle. Tube expansion ratio during bulge forming is 1.22-1.32.The thickness of the bottle became between 0.16 and $0.32 \mathrm{~mm}$. In the experiments, the bottle with 
only an outer cylinder was fabricated, and the specimens are not a double cylinder bottle. This is because the buckling deformation occurs at the outer cylinder only. We also confirmed that the existence of the inner cylinder does not affect the buckling load of the outer cylinder in the analysis. In addition, it is difficult to prepare and to conduct the test with the double wall cylinder. Therefore, we prepared one-wall cylinder specimens. Table 3 and Figure 3 show the shape and size of the specimen used in this experiment. The specimens E1, E2, and E3 correspond to the B1-b, B2-b, and B3-ain analytical model, respectively. Both ends of the specimen were sealed with the epoxy resin in order to maintain atmospheric pressure inside the bottle. The experiment was conducted three times for each specimen.

Table 3: Dimensions of test specimen.

\begin{tabular}{ccc}
\hline Test specimen & $D[\mathrm{~mm}]$ & $L[\mathrm{~mm}]$ \\
\hline E1 & 56 & 157 \\
\hline E2 & 60 & 200 \\
\hline E3 & 67 & 120 \\
\hline
\end{tabular}

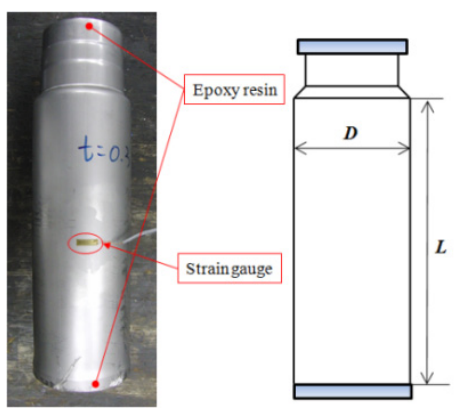

Figure 3: $\quad$ Specimen for air pressure test.

\subsection{Test procedure}

In the experiment, uniform external pressure was loaded to the specimens, and the pressure when the unstable deformation occurred was measured [3]. In order to apply pressure load to specimens, they were installed in the pressurized tank (LIQUID SYSTEM Corporation/SVF-1027) and the inside of a container was pressurized using the air compressor (ANEST IWATA Corporation/CS-175PB). The regulator was used for adjustment of air pouring into the tank, and the pressure sensor (KEYENCE Corporation/ AP-V41A) was used for measurement of the pressure in the tank. In order to measure the deformation of specimens, the strain gauge was glued on the circumferential direction of the central height of the specimen. To improve the sealing property during the strain measurement, the holes for threading codes of strain gauge were sealed by epoxy resin prior to the test. 


\section{Results and discussion}

\subsection{Collapse of bottle}

The deformation of bottle under pressure load was investigated. Figure 4 shows the collapse behavior of the bottle. Figure 4(a) shows the analytical results for Model B2-b (Table 1), and Figure 4(b) shows the experimental results for Model E2.

Both models have the same dimensions. We can see that the out-of-plane displacement of the bottle sharply increased when the pressure reached some value. We defined the critical pressure as the pressure when the strain of bottle increased more than $0.1 \%$. Figure 5 shows the appearance of the bottle after the collapse. The cylindrical bottle became square shaped under pressure conditions.
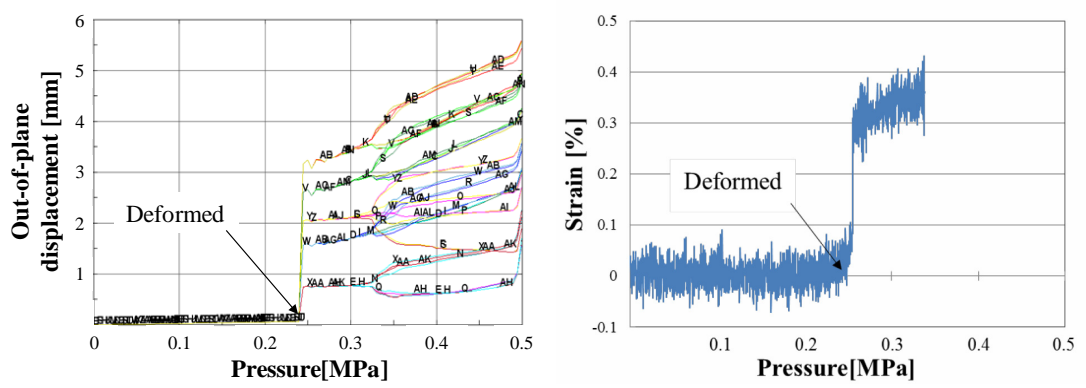

Figure 4: Deformation behavior of bottle induced by external pressure on (a) analytical result (Model B2-b) and (b) experimental result (specimen E2).

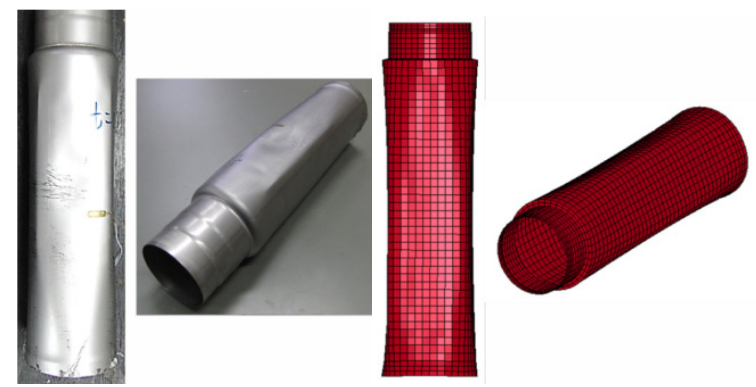

Figure 5: Deformation shape under external pressure on (a) Model B2-b of analysis and (b) specimen E2 of experiment.

All specimens and analytical models showed the same deformation tendency as shown in Figures 4 and 5. Outer cylinder deformed to the square shape, and inner cylinder did not deform for all models. A compression stress is loaded for outer cylinder under pressure loading. In contrast, a tension stress is applied to the inner cylinder. Therefore, buckling of the inner cylinder did not occur. It is 
worth noting that the inner cylinder can be designed in the range of yield strength of the material. As mentioned in section 3.2, the existence of inner cylinder did not affect the buckling strength of the outer layer.

\subsection{Experimental and analytical results}

Figure 6 shows the relationships between thickness and critical pressure of the bottle. It was calculated that critical pressure increased with increasing the thickness of the bottle. Stiffness of the bottle increased with increasing the thickness. Therefore, critical pressure, i.e., the buckling strength of the bottle, improved. Based on a comparison of B1 and B2, or B1-a and B1-b, the diameter and height of bottle affect the critical pressure of the bottle. The critical pressure decreases with increasing diameter or height of the bottle.

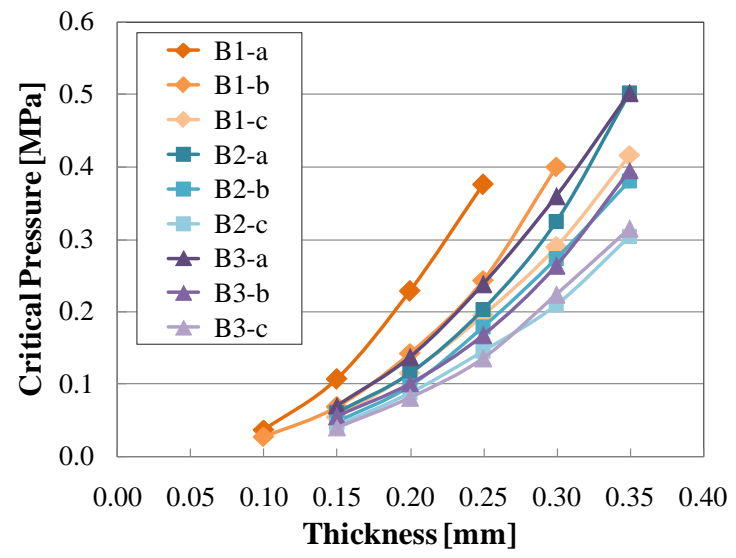

Figure 6: Thickness vs. critical pressure for various models.

The comparison of experimental results and analytical results is shown in Figure 7. The dimension of analytical Models B1-b, B2-b, and B3-a is the same value with the experimental specimens E1, E2, and E3, respectively. Analytical results show good agreement with the experimental results.

To discuss the accuracy of the analysis, the models that have the same thickness with specimens were created, and the analysis was performed. Table 4 shows the comparison between analytical results and experimental results for critical pressure. An average error was $6.2 \%$, and maximum error was $17 \%$ in this comparison. The errors between analytical and experimental results are generated by the defects of the specimens [4]. The cross-section of actual bottle is not a perfect circle. The thickness of the bottle is not completely uniform. In addition, there is residual stress during the fabrication process for the actual bottle. We did not consider these factors in this analysis. It seems that the error depends on the manufacturing process of the bottle. The error of $17 \%$, which is the maximum value in this study, was observed. This error should be considered for the safety design of the bottle. 


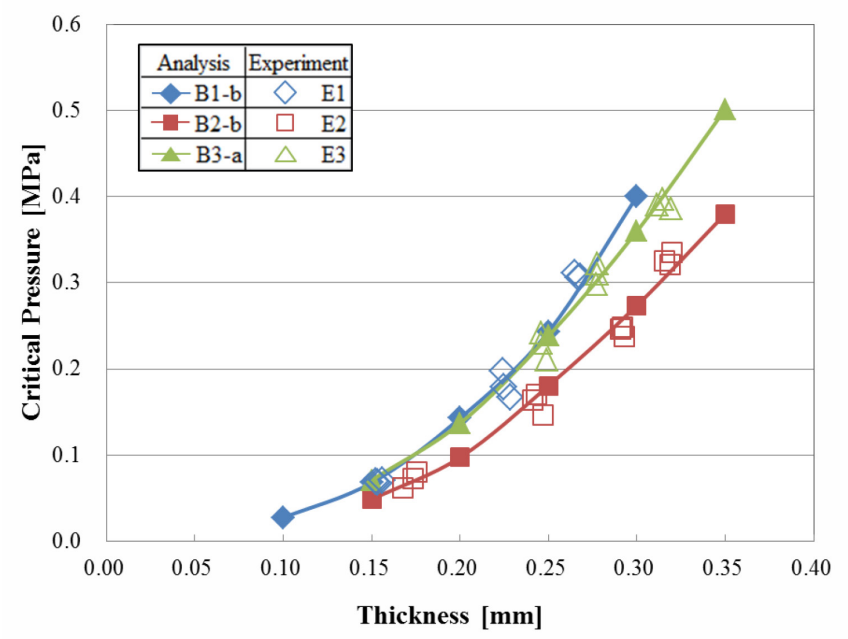

Figure 7: Comparison between experimental and analytical results.

Table 4: Difference between the experiment and the analysis values.

\begin{tabular}{|c|c|c|c|c|c|}
\hline & & \multirow{2}{*}{$\begin{array}{l}\text { Thickness of } \\
\text { specimen } \\
{[\mathrm{mm}]}\end{array}$} & \multicolumn{2}{|c|}{ Critical pressure [MPa] } & \multirow[b]{2}{*}{ Error \% } \\
\hline & & & $\begin{array}{l}\text { Experiment } \\
\text { (E2) }\end{array}$ & $\begin{array}{l}\text { Analysis } \\
\text { (B2-b) }\end{array}$ & \\
\hline \multirow{3}{*}{$\begin{array}{c}\text { Thickness of } \\
\text { steel plate } \mathrm{t}=0.4 \\
\mathrm{~mm}\end{array}$} & 1 & 0.320 & 0.345 & 0.316 & 8.4 \\
\hline & 2 & 0.316 & 0.335 & 0.308 & 8.8 \\
\hline & 3 & 0.319 & 0.332 & 0.314 & 5.4 \\
\hline \multirow{3}{*}{$\begin{array}{c}\text { Thickness of } \\
\text { steel plate } \mathrm{t}= \\
0.35 \mathrm{~mm}\end{array}$} & 1 & 0.293 & 0.237 & 0.261 & 10.1 \\
\hline & 2 & 0.292 & 0.249 & 0.259 & 4.0 \\
\hline & 3 & 0.291 & 0.247 & 0.257 & 4.0 \\
\hline \multirow{3}{*}{$\begin{array}{c}\text { Thickness of } \\
\text { steel plate } \mathrm{t}=0.3 \\
\mathrm{~mm}\end{array}$} & 1 & 0.241 & 0.164 & 0.162 & 1.2 \\
\hline & 2 & 0.247 & 0.147 & 0.172 & 17.0 \\
\hline & 3 & 0.243 & 0.171 & 0.166 & 2.9 \\
\hline \multirow{3}{*}{$\begin{array}{c}\text { Thickness of } \\
\text { steel plate } \mathrm{t}=0.2 \\
\mathrm{~mm}\end{array}$} & 1 & 0.175 & 0.081 & 0.070 & 13.6 \\
\hline & 2 & 0.173 & 0.072 & 0.068 & 5.6 \\
\hline & 3 & 0.168 & 0.061 & 0.062 & 1.6 \\
\hline
\end{tabular}

\subsection{Design method of a vacuum bottle}

The difference in atmospheric pressure of a double-structured vacuum bottle should just assume $1 \mathrm{~atm}(0.1 \mathrm{MPa})$ at a maximum. The critical thickness $t_{\mathrm{c}}$ is defined as the thickness when the bottle is collapsed under the pressure of $1 \mathrm{~atm}$. Based on the results shown in Figure 6, the critical thickness $t_{\mathrm{c}}$ can be determined. It is well known that the ratios of height/diameter and diameter/thickness of the bottle affect the buckling behavior of the bottle under external pressure conditions [5, 6]. Assembling the results based on these ratios, the critical thickness gives the design index of a vacuum bottle as shown in Figure 8. The curve in Figure 8 is drawn based on the analytical results. The experimental results determined by Figure 7 are also listed as blank plots. 
Analytical results show good agreement with the experimental results. The analytical model used in this study is effective for simulating buckling of the bottle under external pressure conditions.

If the diameter and height of bottle are determined, the thickness of the bottle can be determined from Figure 8. When the shape parameter of the bottle is on the right hand area of the curve in Figure 8, the bottle will collapse under $1 \mathrm{~atm}$. In contrast, the bottle with shape parameter on the left side of the curve will keep its shape under atmospheric conditions. However, as mentioned above, an actual bottle has some defect, and there is some error based on the experimental results. Following the design index with consideration of the error, the unexpected collapse phenomenon during the process of drawing a vacuum layer is possible to avoid.

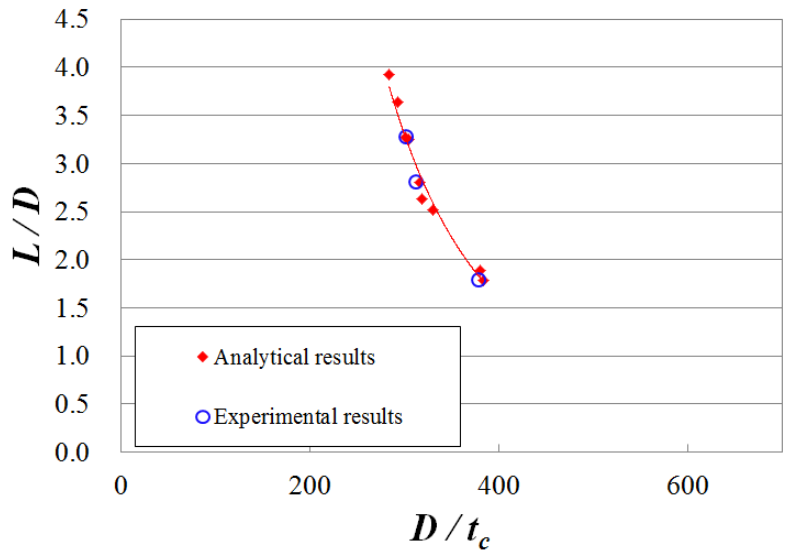

Figure 8: $\quad$ Design index of a vacuum bottle used in this study.

\subsection{Formulation of design index with consideration of material constants}

It is now clear that the design method of the bottle was constructed based on the shape parameter, but this design method would change by the difference of material property that makes up the bottle. Since buckling phenomenon occurs in the elastic deformation, it is clear that this design index is dominated largely by Young's modulus. As shown in Figure 9, this design index is shifted by the difference in Young's modulus. Those materials used in Figure 9 are the materials that may be used in a vacuum bottle, SUS304, Ti-6Al-4V, and Ti. Young's modulus are 197 [GPa], 113 [GPa], and 106 [GPa] respectively. SUS304 is used in Figure 8. Figure 10 plots Young's modulus on a horizontal axis and plots $D / t_{c}$ in case of $L / D=3$ in Figure 9 on a vertical axis. Figure 10 shows the gradient of the linear trend line is calculated as 0.8877 . This gradient represents the dependence of Young's modulus. It is able to formulate the design index by introducing the dependence of Young's modulus to the power approximation.

$$
D / t_{c}=476.08(L / D)^{-0.385}+0.8877(E-197)
$$


By this equation, it was possible to change the buckling strength of the bottle by Young's modulus, which means the design index can be calculated by any new materials, and Young's modulus is able to formulate as a variable number. The second term indicates the amount of shift of the curve by Young's modulus, which is based on SUS304, Young's modulus E=197 [GPa]. However, the shape of the curve is assumed to be unchanged by the difference in Young's modulus. Since this equation is due to shape parameter and Young's modulus, the increase of Young's modulus upper design index, which means it is safer.

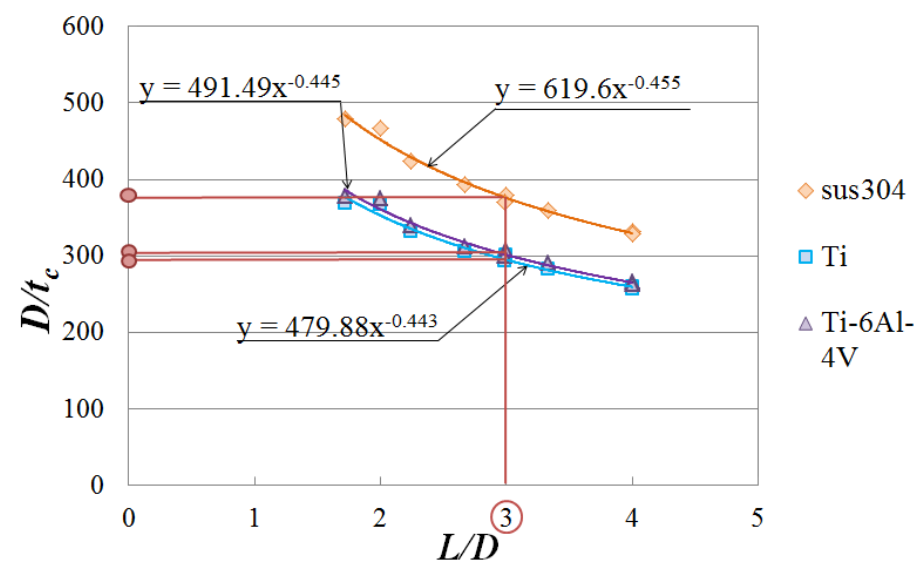

Figure 9: Design index of vacuum bottle using material with a different Young's modulus.

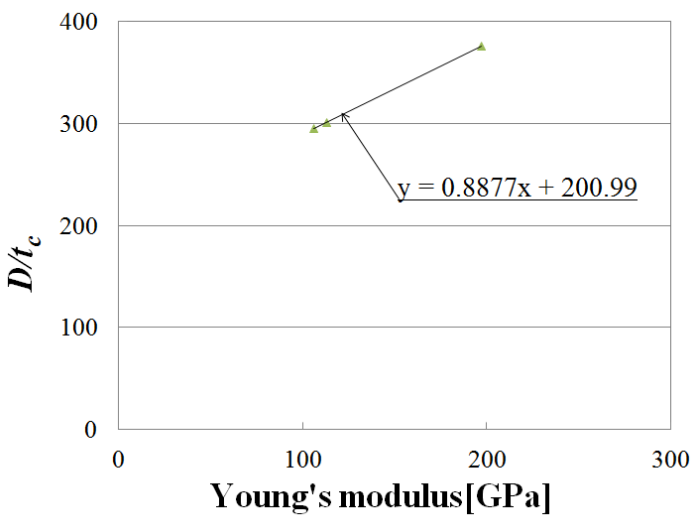

Figure 10: Young's modulus dependence of the ratio of critical thickness to diameter in the case of $L / D=3$. 


\section{Conclusion}

Analytical models of a vacuum bottle were constructed, and the critical pressure that causes the buckling of the bottle was calculated. The critical pressure of the bottle depends on the shape parameter of the bottle. Critical thickness of the bottle was also determined in the analysis. The validity of the analysis was confirmed based on the experiments. Some errors between calculated results and experimental results were observed. The maximum error was $17 \%$, and it seems that this value arises from defects of the bottle during the manufacturing process. Design method of the bottle was constructed based on the shape parameter. The light weight design bottle is possible using the design index suggested in this analysis. And also the shift of index within the increase of Young's modulus can be formulated.

\section{References}

[1] M. Kishi, “The finite-element-method practice handbook for structural analysis”, Morikita Shuppan, 76-88, 2006.

[2] K. Kokubo, H. Nagashima, M. Takayanagi, M. Madokoro, “Japan Society of Mechanical Engineers collected papers (A pieces)”, Vol. 56, No, 532, 193-198, 1990.

[3] S. Kodama, N. Yamaki, "Japan Society of Mechanical Engineers collected papers (A pieces)”, Vol. 49, No, 444, 928-935, 1983.

[4] M. Taneda, "Japan Society of Mechanical Engineers collected papers (The 1st part)” Vol. 26, No, 164, 552-557, 1960.

[5] S. Kuranishi, "The collection of structural mechanics formulas”, Japan Society of Civil Engineers, 400-405, 1986.

[6] H. Kobayashi, "How to use JIS series - The structure and design of pressure vessel”, Japanese Standards Association, 223-226, 2011. 